\title{
Optimized and Enhanced Grid Architecture for Electric Vehicles in Europe
}

\author{
S. ÜBERMASSER ${ }^{1}$, R. RODRÍGUEZ SANCHEZ ${ }^{2}$, C. MADINA ${ }^{2}$, S. BÖCKER ${ }^{3}$, \\ M. GLANCY ${ }^{4}$, E. O'CALLAGHAN ${ }^{4}$, L. SILVESTRE ${ }^{5}$, S. VOIT $^{6}$, G. ODENA BULTÓ ${ }^{7}$, \\ A. GAUL ${ }^{6}$, W. HRIBERNIK ${ }^{1}$ \\ ${ }^{1}$ AIT Austrian Institute of Technology - AUSTRIA \\ ${ }^{2}$ Tecnalia - SPAIN \\ ${ }^{3}$ TU Dortmund - GERMANY \\ ${ }^{4}$ ESB - IRELAND \\ ${ }^{5}$ EDP - PORTUGAL \\ ${ }^{6}$ RWE - GERMANY \\ ${ }^{7}$ ENEL - ITALY
}

\section{SUMMARY}

For an optimized large-scale roll-out of EVs in Europe whilst at the same time maximizing the potential of DER integration, an optimized and enhanced grid architecture for EVs in Europe has to be considered. The work in this paper is addressing this topic and summarizing the corresponding project findings. The aim of this approach is to provide a framework for the further investigation of selected use cases which allows implementing and comparing scenarios of different DSOs. Following a Smart Grid approach, the developed grid architecture implements energy grid entities and ICT components. The general framework was described including all its relevant clusters and indicating related entities. The network types used for this architecture are following the SGAM and Smart Grid Standards Map approach. A so called "Smart Grid Connection Point", which is a generic system interface, is used in this work to allow a more simplified graphical architecture model and increase its readability. Similar to the concept and purpose of the Smart Grid Connection Point, also the principle of an integration bus for entity clusters was introduced. From the Integration bus, the information from/to external systems passes through the Smart Grid Connection Point using one of a range of possible technological options. The position of EVs charging infrastructure within the framework is defined at the border between the domains DERs (generation) and consumption, which takes into account future V2G scenarios, where EVs may act as consumption and generation devices. EVSEs and DERs may be connected as standalone systems directly to the grid, or indirectly as part of one of the clusters at the customer premises domain which refers to the three location-wise types of charging, public, semipublic and private charging. Regarding controlled charging of EVs this optimized architecture allows a variety of different local, distributed or aggregated options which may involve different types of actors.

\section{KEYWORDS}

Smart Grid Architecture, Distributed Generation, Electric Vehicles, Charging Infrastructure 


\section{INTRODUCTION}

The anticipated mass roll-out of electric vehicles (EVs) in Europe and the continuously increasing number of distributed energy resources (DER) are posing major challenges to Europe's Distribution System Operators (DSOs) with regard to ensuring a secure and reliable energy supply and network operation. Despite ongoing research and demonstration activities in this field, particularly the development of new and the revision of existing planning rules and operational principles of DSOs still require extensive R\&D efforts. The overall objective of PlanGridEV [1] is to develop new network planning tools and methods for European DSOs for an optimized large-scale roll-out of EVs in Europe whilst at the same time maximizing the potential of DER integration. For being able to reach this goal, an optimized and enhanced grid architecture for EVs in Europe has to be considered. The work in this paper is addressing this topic and summarizing the corresponding project findings [2]. The high level requirements on "optimized and enhanced grid architecture for electric vehicles in Europe" can be derived from the objectives and vision of the project PlanGridEV (see Figure 1):

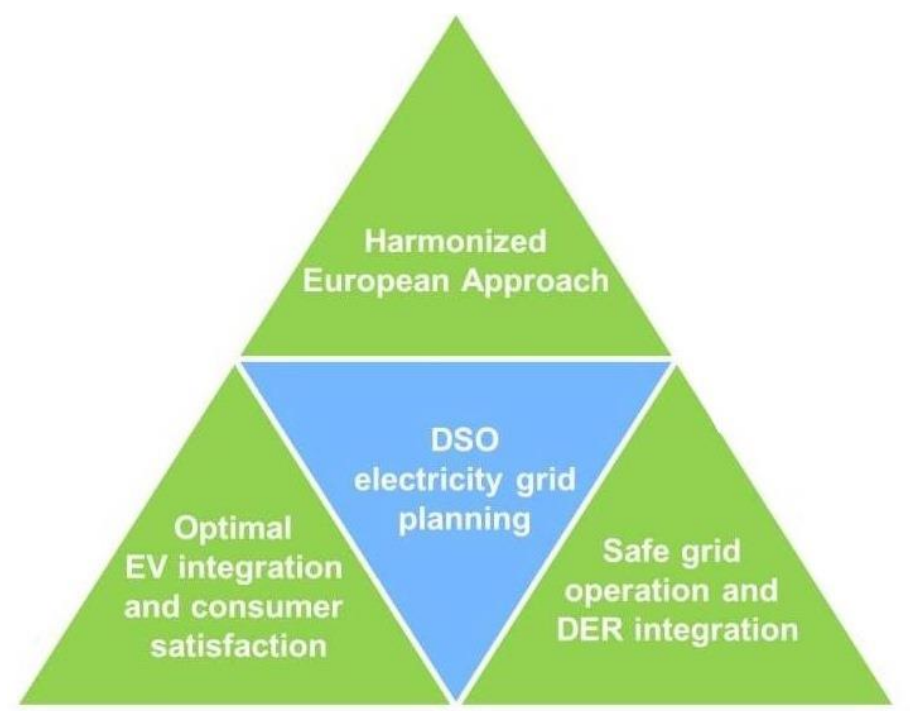

Figure 1 - Main Objectives of PlanGridEV [1]

- Harmonized European approach for the future DSO Smart Grid planning:

This objective emphasizes a harmonized approach especially for the roll-out of EV charging infrastructure but also a vision of a European grid architecture which is applicable within the different areas of Europe and embraces the developments and requirements of expected future scenarios.

- Optimal EV integration and consumer satisfaction:

Solutions for the satisfaction of customer needs while simultaneously allowing different (e.g. grid optimized) charging modes within the capabilities of OEMs (and EVs) is one of the main objectives and challenges of this project.

- Safe grid operation and DER integration:

This includes the development of cost-efficient charging solutions which allow an optimized linkage between DERs and EVs to ensure a maximum in stability of grid operation and a minimum of unnecessary investments.

Additional to the PlanGridEV objectives, smart grid strategies that can be taken into account in a network planning process are classified in four categories [3]:

- Distribution Automation

- Advanced metering infrastructure

- Distributed energy resources

- Customer empowering. 


\section{FRAMEWORK}

This section describes the Smart Grid Architecture Model (SGAM) which is used for the work in PlanGridEV as a framework to describe the optimized and enhanced grid architecture for electric vehicles in Europe. The overall concept and descriptions of the different layers, zones and domains are following the official document "Smart Grid Reference Architecture" from the CEN-CENELEC-ETSI Smart Grid Coordination Group (Version: November 2012) [4].

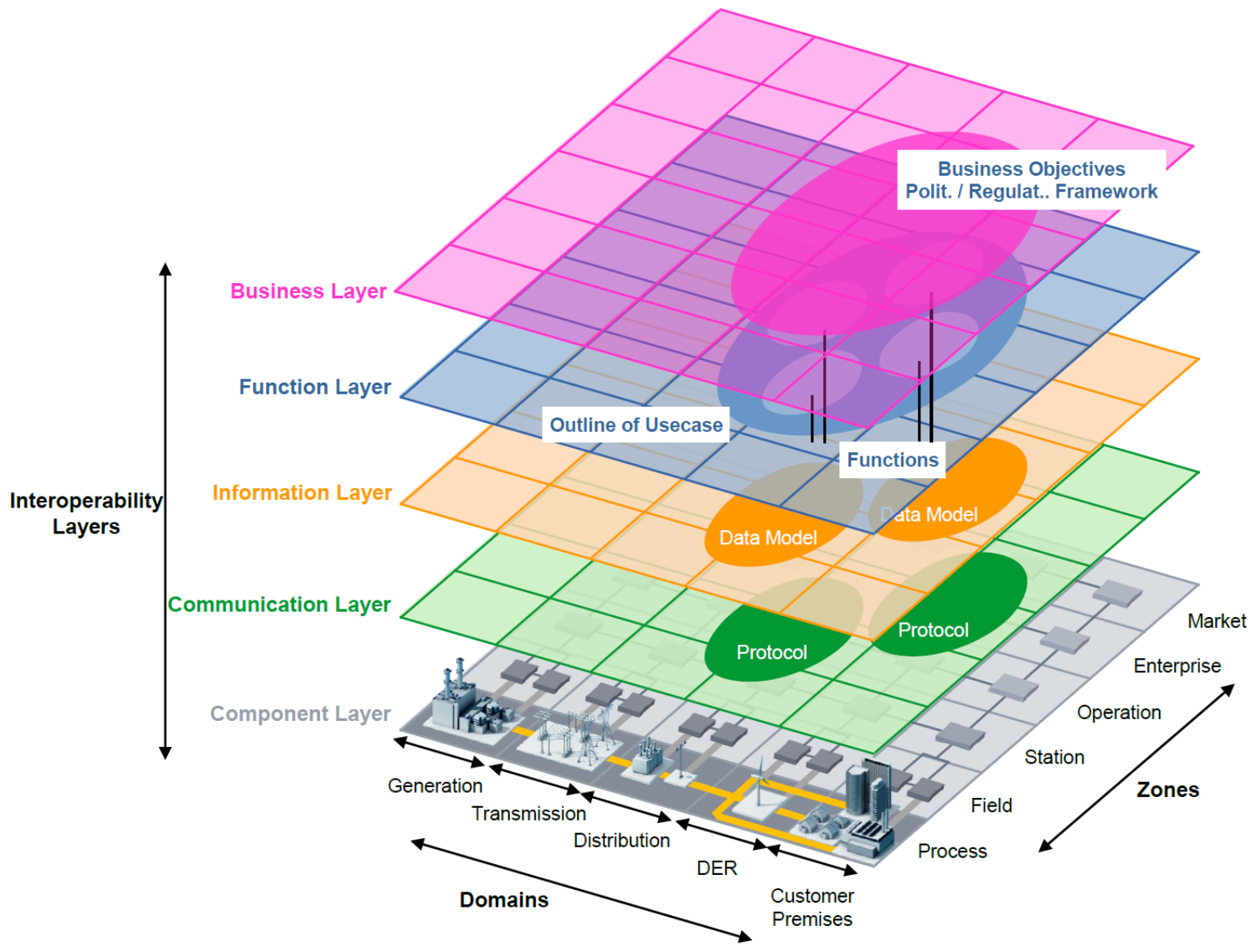

Figure 2 - Smart Grid Architecture Model [5]

The SGAM framework (see Figure 2) and its methodology are intended to present the design of smart grid use cases in an architectural viewpoint allowing it to be specific but also neutral regarding the solution and technology. In accordance to the present scope of the M/490 program, the SGAM framework allows the validation of smart grid use cases and their support by standards. The SGAM framework consists of five layers representing business objectives and processes, functions, information exchange and models, communication protocols and components. These five layers represent an abstract and condensed version of the interoperability categories. Each layer covers the smart grid plane, which is spanned by electrical domains and information management zones. The intention of this model is to represent on which zones of information management interactions between domains take place. It allows the presentation of the current state of implementations in the electrical grid, but furthermore to depict the evolution to future smart grid scenarios by supporting the principles universality, localization, consistency, flexibility and interoperability.

Scenarios and functionalities of Smart Grids rely on full integration of ICT. Table 1 explains the selected communication network types for the optimized and enhanced grid architecture. The classification of the different network types is following the approach of IEC [3]. 
Table 1 - Network Types

\begin{tabular}{|c|l|l|}
\hline Symbol & Network & \multicolumn{1}{c|}{ Description } \\
A & $\begin{array}{l}\text { Subscriber } \\
\text { Access } \\
\text { Network }\end{array}$ & $\begin{array}{l}\text { Networks that provide general broadband access (including but not } \\
\text { limited to the internet) for the customer premises. They are usually not } \\
\text { part of the utility infrastructure and provided by communication service } \\
\text { providers, but can be used to provide communication service for Smart } \\
\text { Grid systems covering the customer premises like Smart Metering and } \\
\text { aggregated prosumers management. }\end{array}$ \\
\hline C & $\begin{array}{l}\text { AMI } \\
\text { Backhaul } \\
\text { Network }\end{array}$ & $\begin{array}{l}\text { Networks at the distribution level upper tier, which is a multi-services } \\
\text { tier that integrates the various sub layer networks and provides backhaul } \\
\text { connectivity in two ways: directly back to control centers or directly to } \\
\text { primary substations to facilitate substation level distributed intelligence. } \\
\text { It also provides peer-to-peer connectivity or hub and spoke connectivity } \\
\text { for distributed intelligence in the distribution level. This network may } \\
\text { serve Advanced Metering or Distribution Automation types of services. }\end{array}$ \\
\hline H & $\begin{array}{l}\text { Backbone } \\
\text { Network }\end{array}$ & $\begin{array}{l}\text { Inter-enterprise or campus networks, including backbone Internet } \\
\text { network, as well as inter-control center networks. }\end{array}$ \\
\hline L & $\begin{array}{l}\text { Operational } \\
\text { Backhaul } \\
\text { Network }\end{array}$ & $\begin{array}{l}\text { Networks that can use public or private infrastructures, mostly to support } \\
\text { remote operation. They usually inter-connect network devices and/or } \\
\text { subsystems to the Operation level over a wide area (region or country). }\end{array}$ \\
\hline
\end{tabular}

For the work in this paper, a "Smart Grid Connection Point" (SGCP), which is a generic system interface, is used. The main reason for its introduction is to allow a more simplified graphical architecture model and increase its readability. The SGCP is defined in detail in SG-CG M490-E [5] and can be described as a conceptual model which defines the physical and logical borderline or interface between different clusters. This might be from the customer to the network/market or from the network/market to the customer considering small scale generation, storage or demand. In the real world the SGCP can be implemented by one or more separate interfaces (e.g. Smart Metering Gateway to an external actor) comprise all new energy services which stem from marketing flexibilities etc.

For this work, the SGCP is used as an abstraction to reduce the overall complexity of the model.

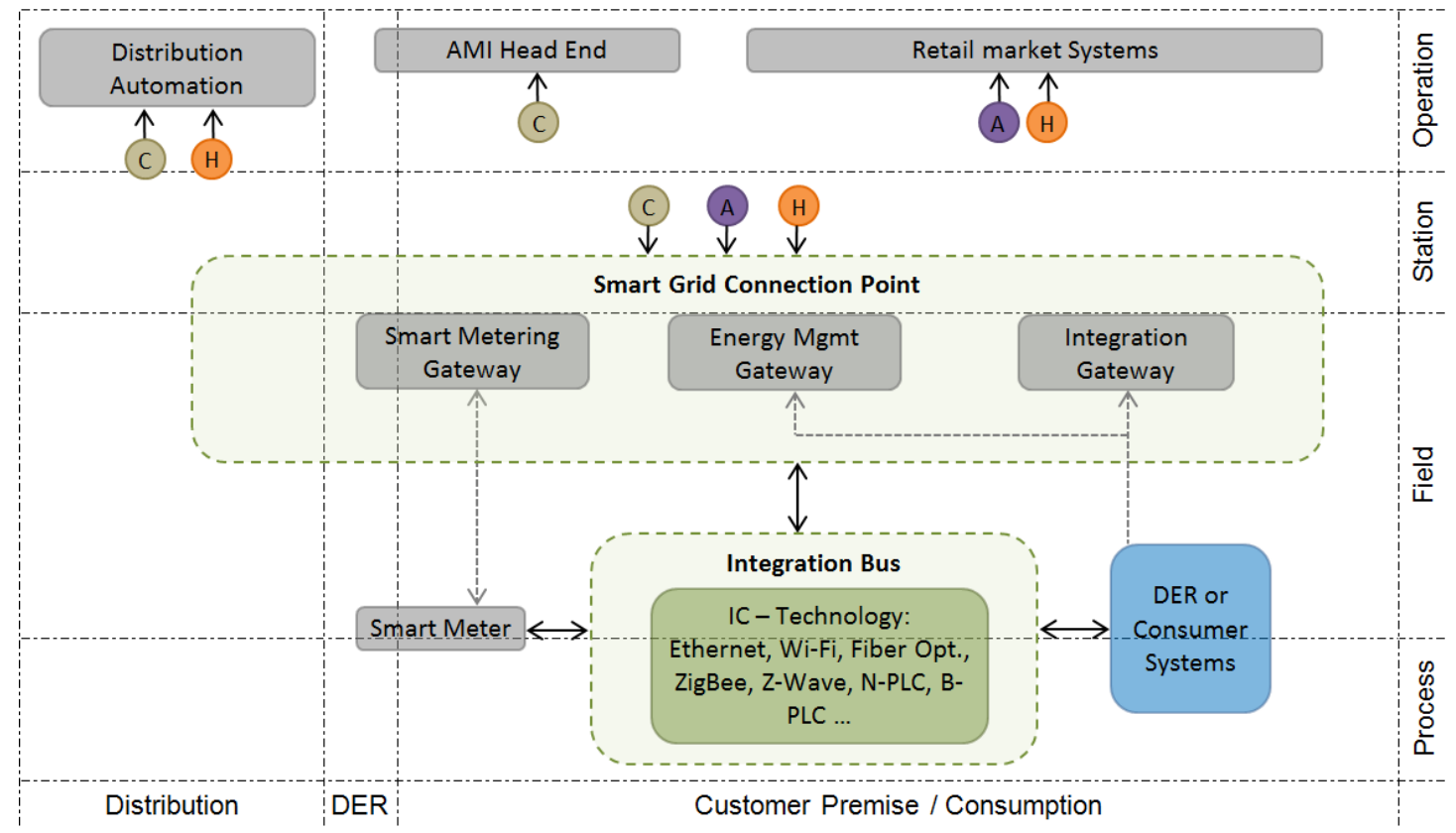

Figure 3 - Graphical example and functional description of SGCP and integration bus 
Similar to the concept and purpose of the SGCP, also the principle of an integration bus for entity clusters was introduced. Figure 3 displays an exemplary integration bus. For increasing the readability and simplification of the overall architecture model, this concept was introduced, following the approach from IEC [6]. In respect of the requirements of this work, the integration bus can be defined as the following: "The integration bus interconnects communication components and sub-systems to form a cluster specific management system (or sub-system)."

\section{OPTIMIZED GRID ARCHITECTURE}

The architecture model developed for PlanGridEV is a generic approach in respect to the Smart Grid Architecture Model [4]. The aim of this approach is to provide a framework for the further investigation of selected use cases which allows implementing and comparing scenarios of different DSOs. Following a Smart Grids approach, the developed grid architecture implements energy grid entities and ICT components. Figure 4 shows the complete Smart Grid plane and entity clusters following the principles of the Smart Grid Standards Map. This figure also includes the main enterprises (the purple colour indicates their allocation to the business layer).

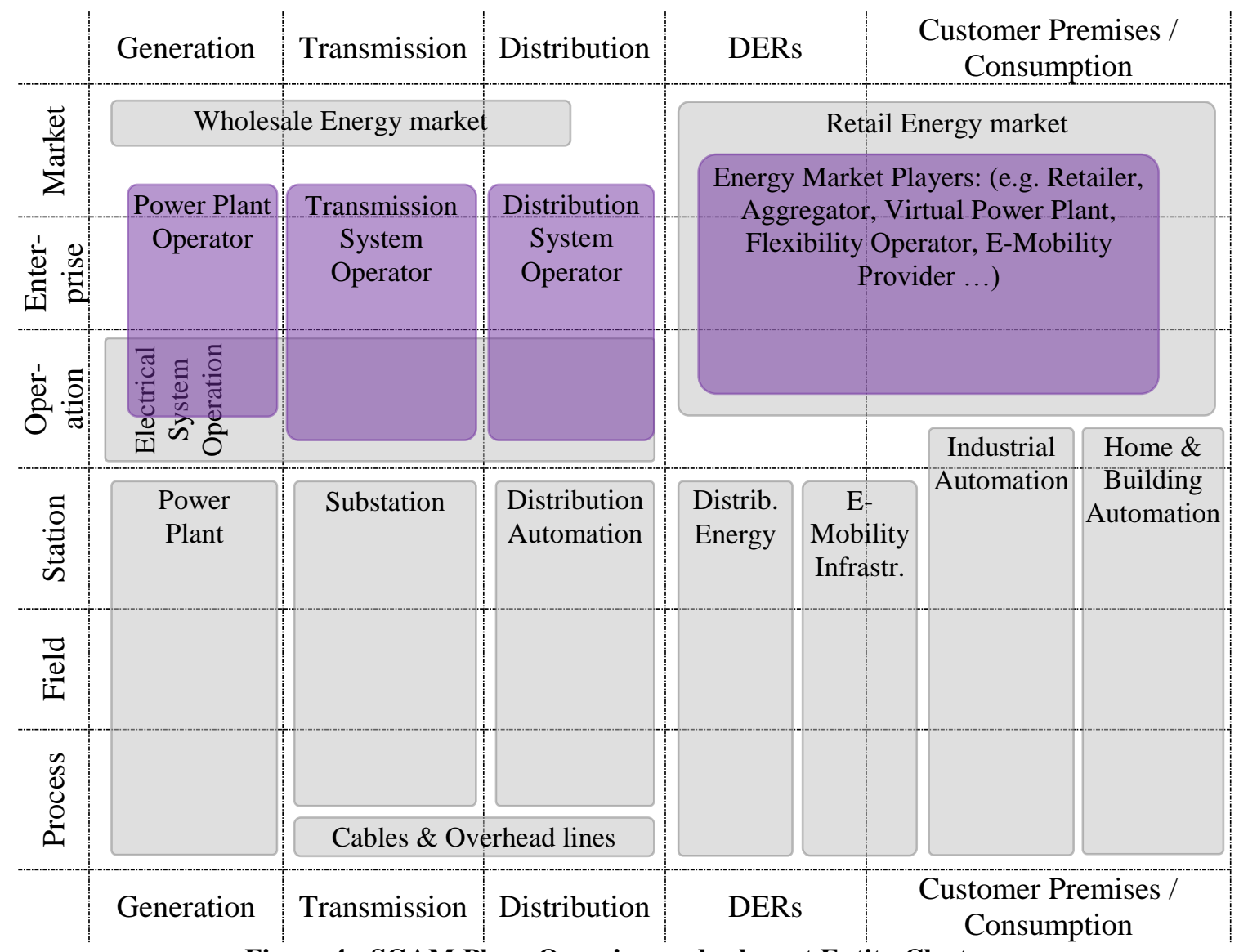

Figure 4 - SGAM Plane Overview and relevant Entity Clusters

Since this architecture focuses on an optimized situation in the future, clusters within the lower levels of the grid are considered to be partly or fully automated. This is also reflected by the naming of some of the clusters (e.g. Industrial or Home \& Building Automation). For increasing the readability, interconnections ICT network are reduced to network type interfaces. Since the specific ICT configuration depends on the scenarios and use cases, this approach provides the necessary flexibility to address specific configurations. The e-mobility infrastructure is located on the border between DERs and the consumption domain. Since EVs are considered to be not only consumers but also generators $(\mathrm{V} 2 \mathrm{X})$ the allocation of this cluster to this position is suitable. 
Figure 5 provides an overview of an approach for mapping different flexibility services within the SGAM, distinguishing between technical, commercial and self-optimization use cases. It also indicates potential users and providers of such services. In respect to the involvement of EVs for providing flexibility services, this representation implies the option for the participation in each of these use cases. In general (but with exceptions) it can be stated, that self-optimization and technical use cases have higher priority than commercial use cases and only remaining flexibilities of entities and clusters may be available for markets.

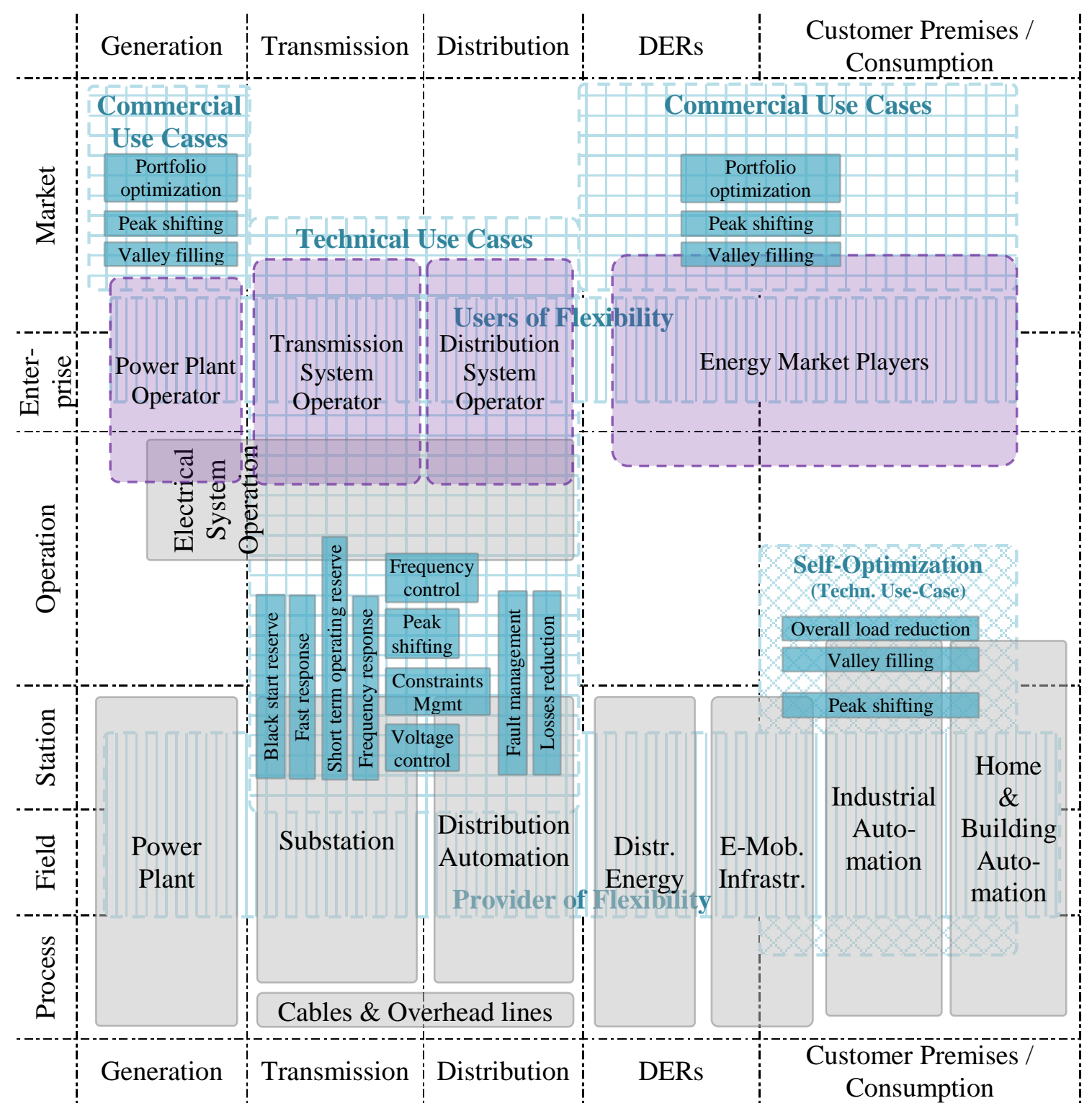

Figure 5 - Mapping of providers and users of flexibility use cases to SGAM

Providers of flexibilities are located within all domains in the zones fields or station (horizontal striped area), whilst users of flexibilities are at the enterprise zone (vertically striped area). Commercial use cases are located at the market zone, addressing the domains generation, DERs and consumption (plaid areas). Technical are by definition and in reference to [7] located at transmission and distribution domains, including substations (also plaid area). The self-optimization use case refers to the consumption domain (including all entity clusters within this domain). This use case can be implemented at different zones, from process to operation (sloped plaid area) and represent therefor the area within the Smart Grid where Flexibility can be used at the most local level. Overall, provision, usage and use cases of flexibility affect all domains and zones of the Smart Grid Plane. 
The optimized and enhanced architecture for EVs in Europe (see Figure 6) is a generic approach following the SGAM framework, the IEC Smart Grid Standards Map and project internal and external findings. Due to the specific focus of the Project PlanGridEV on distribution networks, the architecture shown in Figure 6 only focuses on the following three domains:

- Distribution

- Distributed Energy Resources

- Customer Premises / Consumers

These Domains, contain the following entity clusters:

The home and building automation cluster is located in the domain of customer premises and consumption. Besides typical households, it may also include apartment buildings or functional buildings. This cluster can implement entities from other clusters like EVSE from the E-Mobility infrastructure cluster or DERs or Storage from the DER cluster. Local automation and optimization can be realized and controlled by relevant management systems. Such management systems may monitor, optimize or control according to local (or internal) premises or according to 3rd party objectives. Such information is communicated over the SGCP.

The industrial automation cluster is located in the domain of customer premises and consumption. This cluster covers the area of industrial and larger commercial customers. Although it is very similar to the home $\&$ building cluster, it contains additional entities like load control and process automation, which can be part of the overall management system of a customer at this cluster.

The e-mobility infrastructure cluster is located at the border between the domains consumption and DERs, this is in respect to future scenarios where EVs are considered to act as consumers as well as generators (V2G concepts).

The cluster distributed energy resources focus at small units which generate energy which are connected to medium or low voltage networks. The definition of DERs includes, besides renewable energy resources (e.g. PV systems or wind turbines), also storage devices. DERs can be connected directly to the distribution grid or indirect via the consumption domain at the industrial or home \& Buildings Automation cluster and controlled either by consumers or players at the energy market.

The cluster of distribution automation (DA) includes technologies such as sensors, processors, communication networks, and switches that can perform a number of distribution system functions depending on how they are implemented. In the past, utilities have been applying DA to improve reliability, service quality and operational efficiency. More recently, DA is being applied to perform automatic switching, reactive power compensation coordination, or other feeder operations/control.

The cluster of the distribution system operators and system operation includes mainly the operational aspects and the role of the DSO who is responsible for the safe and secure operation and management of the distribution system.

A retail electricity market exists when end-use customers can choose their supplier from competing electricity retailers. This includes the involvement of flexibility. In contrary to the DSO cluster, where the role of the enterprise is defined very specifically [2] according to the operational tasks, the role of energy market players may vary depending on its relation to the customer. A basic commonality at the operational zone can be found in the management systems, which aggregate either generation and/or consumption. 


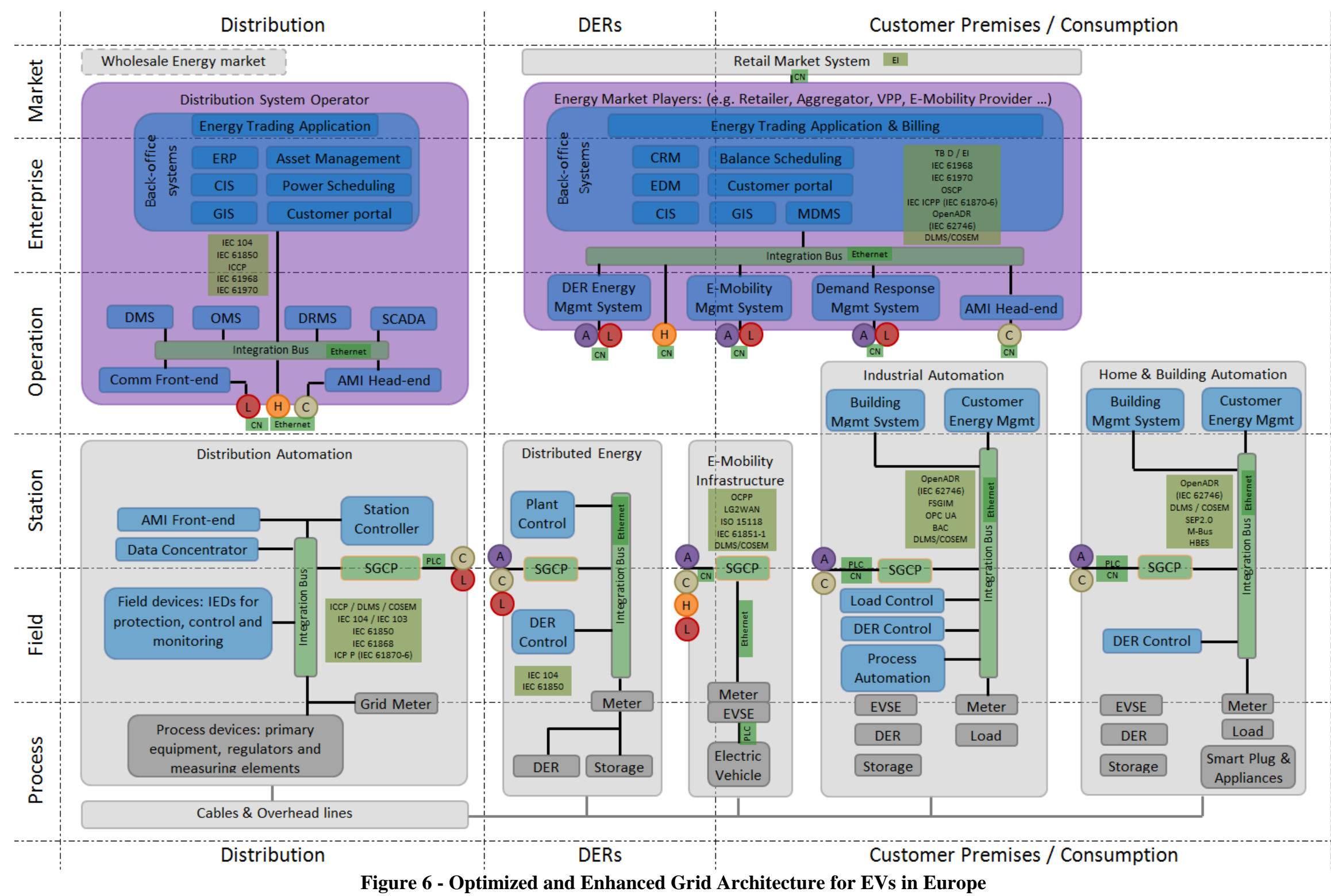


According to the OEM workshop commitments and [8], five situations shall be considered as DSO optimization scenarios; those situations are seen from customers' perspective. The first two use cases controlled and uncontrolled charging cover the whole field of the charging managements or charging strategies and can be combined with the use cases regarding the location of the charging infrastructure (public, semi-public and private). Figure 7 displays the mapping of the OEM use cases within the developed architecture model. The cluster for e-mobility infrastructure is located on the border between the domains Consumption and DERs. This addresses future scenarios, where EVs may act not only as (controllable) consumers but also as generators (where they inject energy to the grid).

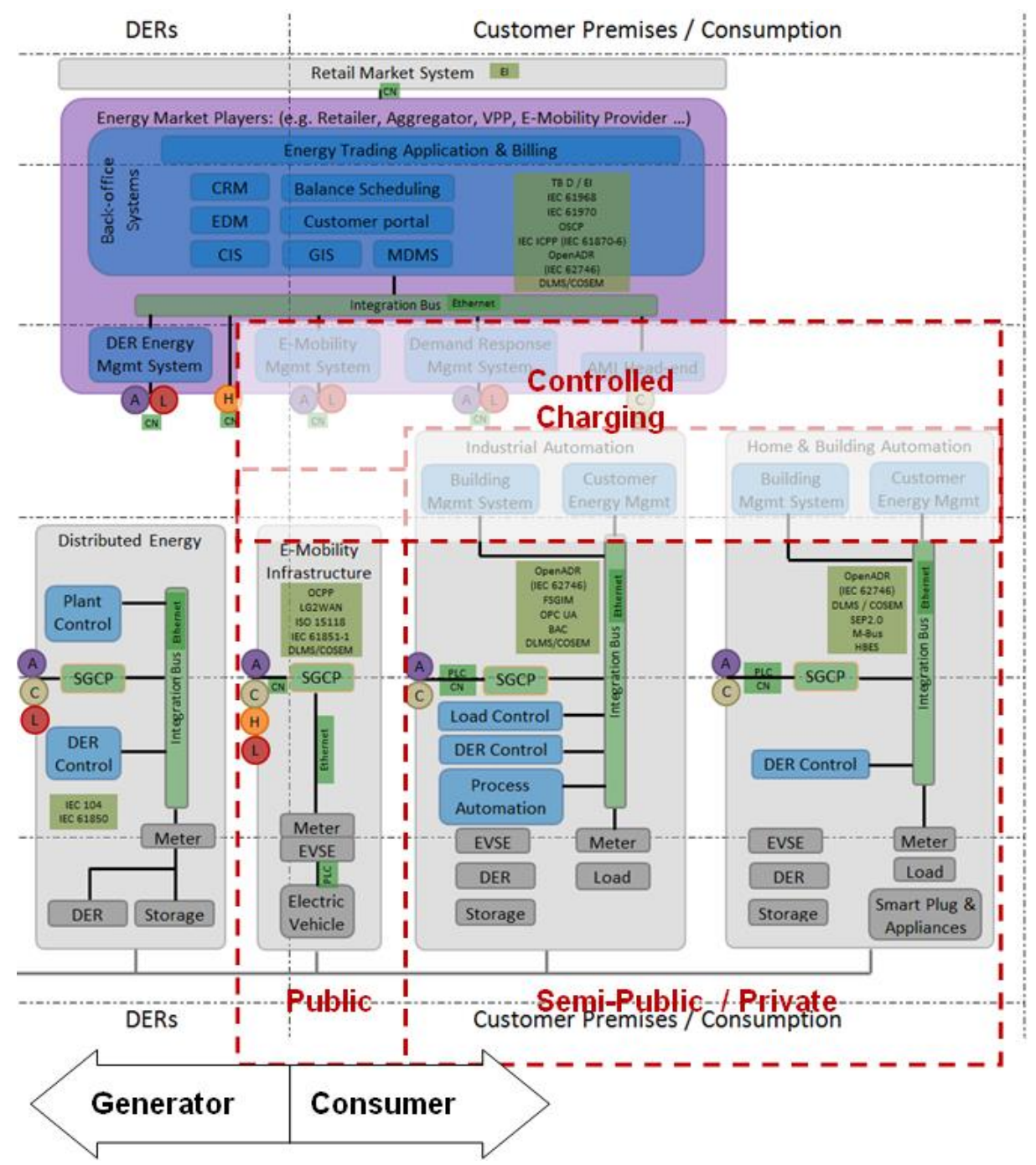

Figure 7 - Optimized Architecture and OEM use cases

Infrastructure for EVs (EVSE) can be implemented within the clusters of home (private charging) and industrial automation (semi-public charging), but it can also be a stand-alone component for public charging. Depending if the charging infrastructure is connected to a management system, controlled charging can be performed besides uncontrolled charging, where only the maximum available power connection and the EV on-board charger decide about the charging profile. Management systems can be part of the home or industrial automation cluster, where decisions regarding charging activities are decided based on local conditions. These local energy management systems (or agents) can also be connected, via the SGCP and the subscriber access network to more high-level and aggregated management systems (e.g. E-Mobility management system or demand response system) and follow its target definitions (this includes also fleet management). This may also apply for public charging infrastructure, with exception of high power charging infrastructure (> 22kW [6], AC and DC), where controlled charging would be contradictorily. 


\section{CONCLUSION \& OUTLOOK}

This paper provides a detailed description of the optimized and enhanced architecture for EVs in Europe as defined within the European project PlanGridEV, which is a generic approach following the SGAM framework, the IEC Smart Grid Standards Map and project internal and external findings. The aim of this approach is to provide a framework for the further investigation of selected use cases, which allow for example the implementation and comparison of scenarios from different DSOs. Following a Smart Grids approach, the developed grid architecture implements energy grid entities and ICT components. Since local conditions of network topology, entities, clusters, number and types of roles ${ }^{1}$, actors ${ }^{2}$ or parties ${ }^{3}$ may differ among countries in Europe, this approach does not claim to be applicable in similar extent within all areas and must therefore be considered as a high-level description.

The general framework was described including all its relevant clusters. Entities within clusters may occur in different composition, depending on the specific scenario. The network types used for this architecture are following the SGAM and Smart Grid Standards Map approach. However, for this work their number is limited to a few selected types, to increase the readability of the architecture by focusing on the most important networks only. Interfaces or connection points (e.g. via the SGCP) at clusters and interconnections to other clusters are depending on the scenario description. Similar to the SGCP, which represents the interface between clusters, for the intra-cluster ICT the concept of an integration bus is introduced. The introduction of a SGCP and integration bus also simplifies the implementation process of use cases and scenarios since complex ICT architectures are reduced to a minimum. In contrary to the Smart Grid Standards Map, advanced metering infrastructure is not represented by an own cluster.

The architecture considers and implements all four categories of the Smart Grid Strategies. Automation on the distribution level, but also at the consumption domain, is part of this approach, as is the integration of DERs. The empowerment of customers is reflected in several perspectives. Clusters at the consumption domain are considered to feature a certain level of automation and can implement systems from DER or e-mobility infrastructure clusters. Additionally the SGCP interface allows communication to external parties (e.g. market players) and forwarding relevant information regarding flexibilities remaining (optional after potential self-optimization management procedures). With exception of the advanced metering infrastructure (AMI), these categories are directly represented within the architecture as specific clusters or domains. The AMI is considered to be an integrated part of the architecture and is distributed and implemented within all relevant clusters.

The position of EVs charging infrastructure within the framework is defined at the border between the domains DERs (generation) and consumption, which takes into account future V2G scenarios, where EVs may act as consumption and/or generation device. EVSEs and DERs may be connected as standalone systems directly to the grid, or indirectly as part of one of the clusters at the customer premises domain which refers to the three location-wise types of charging, public, semi-public and private charging. Regarding controlled charging of EVs this optimized architecture allows a variety of different local, distributed or aggregated options which may involve different types of actors (e.g. market players).

Since the architectures aim is to function as a framework for investigating different approaches and scenarios, this model does not imply a specific optimization hierarchy or strategy. However, from the entities at the cluster it can be derived that most domains contain multiple levels of optimization

\footnotetext{
${ }^{1}$ Role: it represents the external intended behaviour (i.e. a responsibility) of a party. They describe external business interactions with other parties in relation to the goal of a given business transaction.

${ }^{2}$ Actor: an actor represents a party that participates in a business transaction. Within a given business transaction an actor performs a task in a specific role or a set of roles. An actor is a composition of one or more roles.

${ }^{3}$ Party: parties are legal entities, i.e. natural persons or organizations. They can bundle different roles according to their business model.
} 
options distributed across different zones. This considers and allows different optimization strategies which can be centralized or more distributed approaches. This also includes optimization and automation at the lowest levels of the network hierarchy.

Flexibility services can be mapped to this architecture and clustered within specific use cases. In general, commercial and technical use cases can be distinguished. Whilst commercial services are addressed by markets, only some of flexibility services in the technical use case are market products and the rest are fully automated functions (e.g. fault management) at the zones field or station within the domains distribution and/or transmission. Additionally to these two use case clusters, a third field of applications for flexibility can be identified which is expected to increase in importance in future. Self-optimization at the consumption domain, including DERs (incl. stationary storage systems) and emobility infrastructure is related to the technical use cases, since involved systems will follow the signals of local management systems. However, the reasons and strategies for optimizing can also be of commercial nature. Overall, provision, usage and use cases of flexibility affect all domains and zones of the Smart Grid Plane.

The cluster of retail energy market contains the entity cluster within the enterprise zone for a variety of different parties. This may include retailers, aggregators, VPPs, flexibility providers or others. For the management of aggregated flexibilities of relevant entities by actors several systems are part of the retail market cluster at the operational zone. At the current version, the architecture contains DER, mobility and DR management systems, which possibly have to be expanded by additional systems in future.

\section{ACKNOWLEDGMENTS}

The research leading to these results has received funding from the European Union Seventh Framework Programme (FP7/2007-2013) under grant agreement No. 608957.

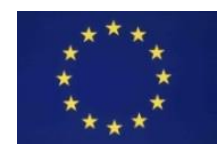

\section{BIBLIOGRAPHY}

[1] "PlanGridEV - Distribution grid planning and operational principles for EV mass roll-out while enabling DER integration." [Online]. Available: http://plangridev.eu/index.html. [Accessed: 12Dec-2014].

[2] Stefan Übermasser, Raúl Rodríguez, Carlos Madina, Stefan Böcker, M. Glancy, E. O'Callaghan, Luís Silvestre, Gemma Odena Bultó, and Stephan Voit, "D6.1 Optimized and Enhanced Grid Architecture for Electric Vehicles in Europe," Project Deliverable D6.1, 2015.

[3] Raúl Rodríguez, Carlos Madina, Eduardo Zabala, Mario Nunes, Luís Silvestre, Susete Albuquerque, Eoghan O'Callaghan, Jonathan Sandham, Armin Gaul, Stefan Willing, and Stephan Voit, "D3.3 New ICT developments and services for EV integration in electricity distribution networks," Project Deliverable D3.3, 2015.

[4] "Smart Grid Reference Architecture," CEN-CENELEC-ETSI Smart Grid Coordination Group, Nov. 2012.

[5] "SG-CG/M490/E - Sustainable Processes," CEN-CENELEC-ETSI Smart Grid Coordination Group, SG-CG/M490/E Version 1.0, Nov. 2012.

[6] IEC, "Smart Grid Standards Map," Smart Grid Standards Map. [Online]. Available: http://smartgridstandardsmap.com/.

[7] "SG-CG/M490/L - Flexibility Management," CEN-CENELEC-ETSI Smart Grid Coordination Group, SG-CG/M490/L Version 3.0, Nov. 2014.

[8] Stefan Übermasser, Johannes Stöckl, Sawsan Henein, Tara Esterl, Luís Silvestre, Stephane Rapoport, Pedro Carvalho, and Raul Rodriguez Snachez, "D1.1 Current requirements, regulatory gaps and expected benefits." PlanGridEV, 2013. 